\title{
Broadband terahertz spectroscopy of the insulator-metal transition driven by coherent lattice deformation at the $\mathrm{SmNiO}_{3} / \mathrm{LaAlO}_{3}$ interface
}

\author{
W. Hu, ${ }^{1, *}$ S. Catalano, ${ }^{2}$ M. Gibert, ${ }^{2}$ J.-M. Triscone, ${ }^{2}$ and A. Cavalleri ${ }^{1,3, \dagger}$ \\ ${ }^{1}$ Max Planck Institute for the Structure and Dynamics of Matter, Center for Free Electron Laser Science, 22761 Hamburg, Germany \\ ${ }^{2}$ Department of Quantum Matter Physics, University of Geneva, CH-1211 Geneva, Switzerland \\ ${ }^{3}$ Department of Physics, Clarendon Laboratory, University of Oxford, OX1 3PU Oxford, United Kingdom
}

(Received 29 February 2016; revised manuscript received 28 March 2016; published 12 April 2016)

\begin{abstract}
We investigate the nonequilibrium insulator-metal transition driven in $\mathrm{SmNiO}_{3}$ thin film by coherent optical excitation of the $\mathrm{LaAlO}_{3}$ substrate lattice. By probing the transient optical properties over a broad frequency range $\left(100-800 \mathrm{~cm}^{-1}\right.$ ), we analyze both the time-dependent metallic plasma and the infrared optical phonon line shapes. We show that the light-induced metallic phase in $\mathrm{SmNiO}_{3}$ has the same carrier density as the equilibrium metallic phase. We also report that the $\mathrm{LaAlO}_{3}$ substrate acts as a transducer only at the earlier time delays, as the vibrations are driven coherently. No long-lived structural rearrangement takes place in the substrate. Finally, we show that the transient insulator-metal transition occurs both below and above the Néel temperature. We conclude that the supersonic melting of magnetic order measured with ultrafast $\mathrm{x}$ rays is not the driving force of the formation of the metallic phase. We posit that the insulator-metal transition may origin from the rearrangement of ordered charges at the interface propagating into the film.
\end{abstract}

DOI: 10.1103/PhysRevB.93.161107

The metal-insulator and magnetic transitions of nickelates $[1,2]$ can be systematically manipulated at equilibrium with interfacial strain. By changing substrate materials and introducing either compressive or tensile strain one can, for example, tune the metal-insulator transition temperature over several hundred Kelvins for the nickelate films.

In the same spirit, we have shown in the past that by optically exciting the lattice of $\mathrm{LaAlO}_{3}$ or $\mathrm{NdGaO}_{3}$ substrates, one could induce ultrafast switching of the electrical and magnetic properties of the nickelate thin film $\mathrm{NdNiO}_{3}$. A longlived, 5-order-of-magnitude increase in the low-frequency conductivity [3] was measured, accompanied by the propagation of a supersonic antiferromagnetic/paramagnetic front [4]. The relationship between substrate excitation, creation of mobile charges, melting of magnetism, and a possible structural rearrangement in the nickelate film is yet to be determined.

First, to date we could not determine if the transient state reaches the same metallic phase as that achieved thermally at equilibrium. Because the transient reflectivity was probed over a limited frequency range (up to $25 \mathrm{meV}, 200 \mathrm{~cm}^{-1}$ ) [3], we could not cover the whole Drude peak in $\mathrm{NdNiO}_{3}$ [5] and the phonon modes in the $\mathrm{LaAlO}_{3}$ substrate [6]. Hence, the total Drude spectral weight in the transient phase could not be retrieved reliably. The experiments reported here show that the nickelate film switches to a state that has the same Drude weight of the equilibrium metallic phase. This effect is, however, also established to be not thermal, as the substrate structural peaks reflect minimal heating.

Second, the role of the substrate lattice in switching the electronic phase in the thin film has been unknown. Indeed, it has not been clear if the lattice of the substrate is undergoing a long-lived structural rearrangement that sustains the metallic phase or if the coupling is only taking place at early times. In the data reported here we show that no rearrangement takes

\footnotetext{
*wanzheng.hu@mpsd.mpg.de

†andrea.cavalleri@mpsd.mpg.de
}

place in the substrate and that the phonon resonances are not modified after the interaction with the pump light.

Third, to date it was not clear if the melting of magnetism observed with ultrafast x-ray scattering [4] causes the lightinduced insulator-metal transition. This cannot be determined easily in $\mathrm{NdNiO}_{3}$, because in this material the insulator-metal transition and the antiferromagnetic ordering temperatures coincide [7]. By measuring in $\mathrm{SmNiO}_{3} / \mathrm{LaAlO}_{3}$, which exhibits a well-separated metal-insulator transition and Néel temperatures [7], we show here that the transient insulatormetal transition can be induced both in the antiferromagnetic and paramagnetic insulators and that melting of magnetism observed in $\mathrm{NdNiO}_{3}$ [4] is not a necessary condition for the ultrafast insulator-metal transition.

$\mathrm{SmNiO}_{3}$ epitaxial thin films (24 unit cells, $10 \mathrm{~nm}$ thick) were used, grown by off-axis radio frequency magnetron sputtering on a (001) $\mathrm{LaAlO}_{3}$ single-crystal substrate [2]. The $\mathrm{LaAlO}_{3}$ substrate was a $5 \times 5 \mathrm{~mm}^{2}$ size, twinned sample with edges parallel to the pseudocubic crystallographic axes.

The equilibrium optical and transport properties of the nickelate heterostructure are shown in Fig. 1. The $a b$-plane reflectivity of the bare substrate and the nickelate heterostructure were measured at near-normal incidence from 100 to $15000 \mathrm{~cm}^{-1}$ using a Bruker vertex $80 \mathrm{v}$ spectrometer. A gold mirror was used as a reference for the reflectivity measurement at various temperatures from 10 to $295 \mathrm{~K}$. As plotted in Fig. 1(a), the reflectivity of the bare substrate is dominated by three square-shaped peaks at around 200,450 , and $700 \mathrm{~cm}^{-1}$ in the far-infrared region. These are infrared-active phonons [8] with strong temperature dependence. In particular, the maximum reflectivity at the $700-\mathrm{cm}^{-1}$ phonon decreases from 0.75 to 0.6 when increasing the temperature from 10 to $295 \mathrm{~K}$. Figure 1(b) is the far-infrared reflectivity of $\mathrm{SmNiO}_{3} / \mathrm{LaAlO}_{3}$. The reflectivity resembles that of the bare substrate, since the overall light penetration depth at far infrared $(\sim \mu \mathrm{m})$ is much deeper than the $\mathrm{SmNiO}_{3}$ film thickness $(10 \mathrm{~nm})$. The rounding off of the flat substrate phonon bands is due to a finite conductivity in $\mathrm{SmNiO}_{3}$, including the free-carrier 


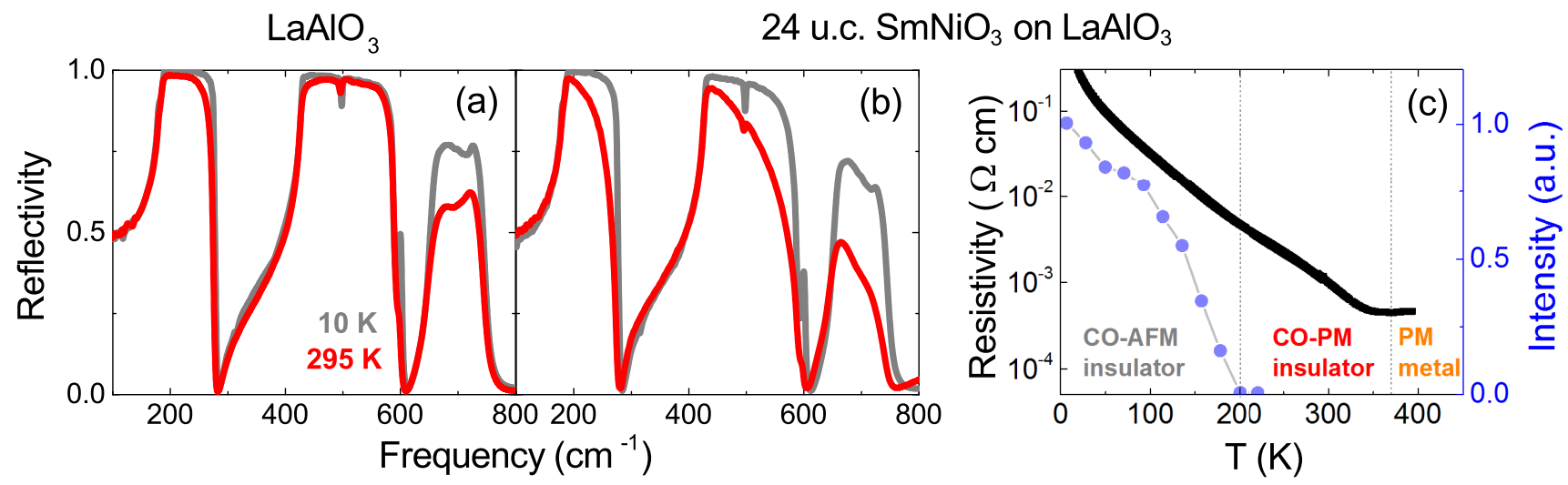

FIG. 1. Equilibrium optical reflectivity of (a) the bare $\mathrm{LaAlO}_{3}$ substrate and (b) the nickelate heterostructure. For both materials, the optical response is dominated by three infrared-active phonons of $\mathrm{LaAlO}_{3}$ at around 200,450 , and $700 \mathrm{~cm}^{-1}$. (c) Temperature dependence of the resistivity (black solid line) and the magnetic diffraction peak intensity (blue circles) [2] for the $\mathrm{SmNiO}_{3}$ thin film. $\mathrm{SmNiO}$ is a paramagnetic (PM) metal above $370 \mathrm{~K}$, a charge-ordered (CO) paramagnetic insulator at $200 \mathrm{~K}<T<370 \mathrm{~K}$, and a charge-ordered antiferromagnetic (AFM) insulator below $200 \mathrm{~K}$.

contribution and the low-frequency tail of the insulating and charge-order gaps [9]. Our optical spectra agree very well with earlier data on $\mathrm{SmNiO}_{3} / \mathrm{LaAlO}_{3}[9,10]$. The insulator-metal transition temperature for our $\mathrm{SmNiO}_{3} / \mathrm{LaAlO}_{3}$ sample is $370 \mathrm{~K}$ from the four-probe resistivity measurement, and the antiferromagnetic ordering temperature is $200 \mathrm{~K}$ as revealed by resonant X-ray diffraction [Fig. 1(c)] [2].

For the pump-probe experiment, we used midinfrared pump pulses to drive the substrate phonon and measured the transient broadband reflectivity at normal incidence with linearly polarized light along the [100] direction [11]. The broadband probe pulses were generated and probed in a laser-ionized gas plasma [12] using 800-nm pulses with 1-mJ energy and 35-fs duration from a Ti:sapphire laser. To measure the high-frequency range with sufficient signal-to-noise ratio, the probe pulses were further detected in a 50- $\mu$ m-thick Z-cut GaSe crystal, which was tilted $\sim 45$ degrees with respect to the incident beam [13]. In this way, we could measure the transient reflectivity from 300 to $800 \mathrm{~cm}^{-1}$, which is crucial for detecting possible structural changes of the infrared-active phonons in $\mathrm{LaAlO}_{3}$.

The midinfrared pump pulses were generated by differencefrequency mixing in a home-built two-stage optical parametric amplifier. The center frequency was tuned to the $750-\mathrm{cm}^{-1}$ longitudinal plasma frequency of the oxygen stretching mode [8] in $\mathrm{LaAlO}_{3}$. Because of the twinned structure of the rhombohedral $\mathrm{LaAlO}_{3}$ and the strong mixing of the phonon eigenvectors in perovskites [14], our pump pulses drove the oxygen stretching mode with mixed $A_{2 u} / E_{u}$ symmetries. The time duration of the pump pulses is $300 \mathrm{fs}$. The pump fluence is $2 \mathrm{~mJ} / \mathrm{cm}^{2}$.

We first studied the transient reflectivity of the bare substrate at $T=10 \mathrm{~K}$. Figure 2(a) plots the equilibrium and transient reflectivity at selected time delays. The transient reflectivity changes, $\Delta R=R^{\text {transient }}-R^{\text {equilibrium }}$, from 0 to 30 ps are shown as color plots in Figs. 2(b) and 2(c). No detectable phonon reshaping or intensity changes can be seen at all measured time delays, indicating the lattice symmetry remains the same as the equilibrium symmetry. Excitation of the substrate lattice, which follows the high-frequency midinfrared field during the first $150 \mathrm{fs}$, does not change on average. By comparing the measured data with the strong temperature dependence of the reflectivity [Fig. 1(a)], we conclude that light-induced heating is negligible.

We measured the transient reflectivity of the $\mathrm{SmNiO}_{3} /$ $\mathrm{LaAlO}_{3}$ heterostructure with the pump pulses polarized either perpendicular or parallel to the probe pulses. In the pump $\perp$ probe case, a further rounding off of the substrate phonon bands with increasing time delays can be seen in Fig. 2(d) and the differential reflectivity plots [Figs. 2(e) and 2(f)]. The transient reflectivity increases (blue)/decreases (red) below/above the transverse plasma frequencies of the substrate phonon modes at 450 and $700 \mathrm{~cm}^{-1}$. The transient reflectivity change reaches maximum at $1-$ ps time delay.

Since experiments in the bare $\mathrm{LaAlO}_{3}$ substrate yield no pump-probe response [Figs. 2(a)-2(c)], the reflectivity changes reported here for the $\mathrm{LaAlO}_{3} / \mathrm{SmNiO}_{3}$ should come from the nickelate thin film. When the pump pulses were polarized parallel to the probe pulses, we observed a transient rounding off of the substrate phonon bands comparable to that seen in the pump $\perp$ probe case. However, a new absorption feature emerged at around $700 \mathrm{~cm}^{-1}$ [Fig. 2(i)], which is close to the transverse plasma frequency of the substrate oxygen stretching mode. This $700-\mathrm{cm}^{-1}$ feature develops after the pump pulses have left, redshifts and then blueshifts, and finally disappears before $1 \mathrm{ps}$, the time when the transient rounding off of the substrate phonon bands reaches a maximum.

The transient reflectivity reported above was fitted with the same model used for the material at equilibrium. In both cases, we fitted the substrate and the thin film separately by Drude-Lorentz terms and then used a two-layer model [15] to fit the reflectivity of the heterostructure,

$$
\tilde{r}_{\mathrm{SmNiO}_{3} / \mathrm{LaAlO}_{3}}=\frac{\tilde{r}_{\mathrm{SmNiO}_{3}}+\tilde{r}_{\mathrm{LaAlO}_{3}} \exp (2 i \delta)}{1+\tilde{r}_{\mathrm{SmNiO}_{3}} \tilde{r}_{\mathrm{LaAlO}_{3}} \exp (2 i \delta)},
$$

where $\delta=2 \pi d\left(n_{\mathrm{SmNiO}_{3}}+\mathrm{i} k_{\mathrm{SmNiO}_{3}}\right) / \lambda$. Here $n_{\mathrm{SmNiO}_{3}}$ is the refractive index and $k_{\mathrm{SmNiO}_{3}}$ is the extinction coefficient of the $\mathrm{SmNiO}_{3}$ layer, $d$ is the film thickness, and $\lambda$ is the wavelength

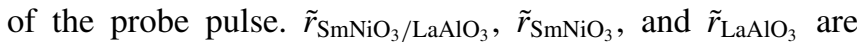




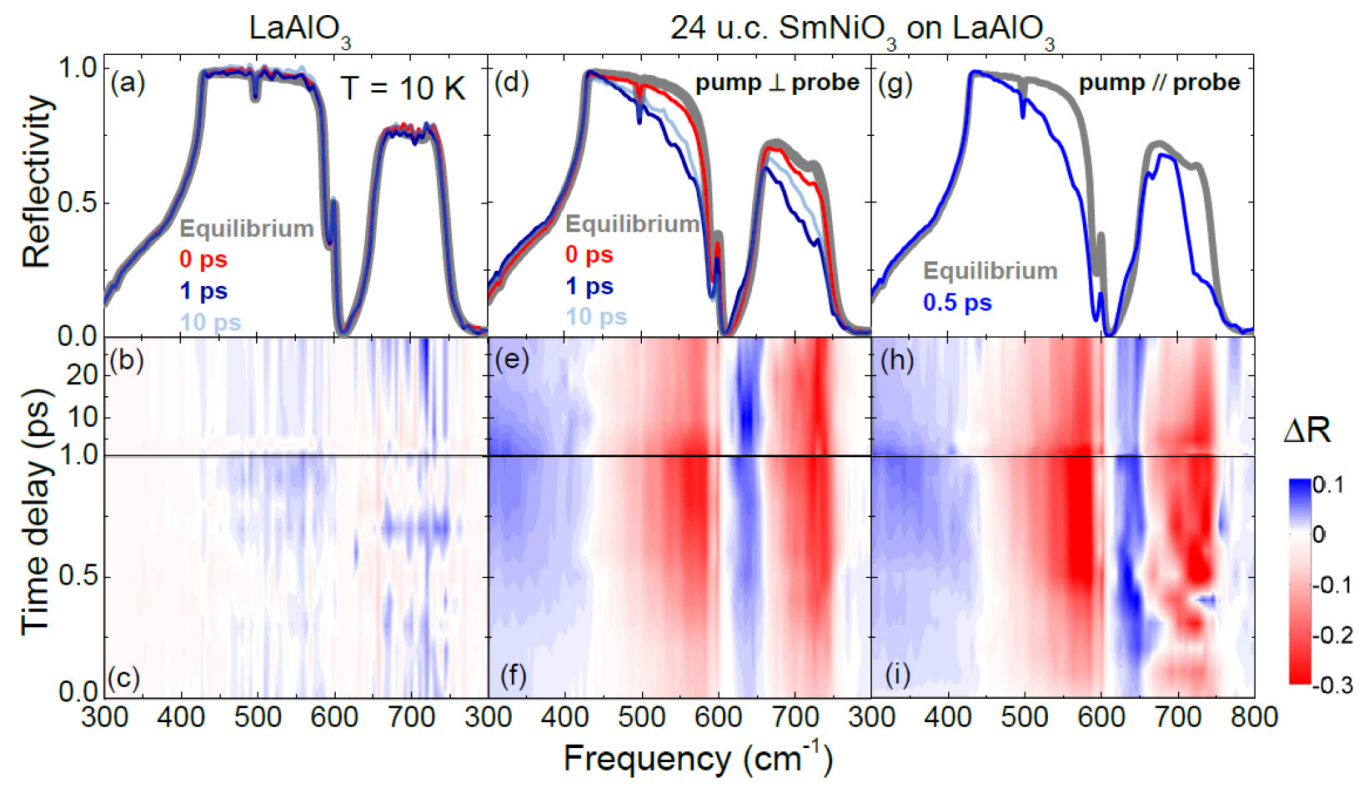

FIG. 2. Transient optical reflectivity of the bare substrate $(a-c)$, and the nickelate heterostructure measured with perpendicular $(d-f)$, and parallel (g-i) pump-probe geometries at $T=10 \mathrm{~K}$. Upper panel: transient reflectivity at selected time delays. Middle and lower panels: transient reflectivity change from 0 to $30 \mathrm{ps}$. The 0 -ps time delay is defined as the peak of the pump pulse.

the reflection coefficients of the heterostructure, the $\mathrm{SmNiO}_{3}$ layer, and the $\mathrm{LaAlO}_{3}$ substrate, respectively.

Because measurements in the bare substrate did not show any light-induced changes, we used the equilibrium fitting parameters for the substrate and time-varying parameters for the nickelate layer to fit the transient reflectivity. The fitting was performed under the assumption of a homogeneous response for the thin film.

For the pump $\perp$ probe case, the transient reflectivity at all time delays was well fitted by changing only the zerofrequency conductivity value in the nickelate layer; for the pump//probe case, extra Lorentzians are used for the nickelate layer to fit the $700-\mathrm{cm}^{-1}$ feature at early delays [Fig. 2(i)]. In the end, we obtained similar zero-frequency conductivity values for the parallel and the perpendicular pump-probe geometries.

Figure 3(a) shows the fitting result for the broadband transient reflectivity of $\mathrm{SmNiO}_{3} / \mathrm{LaAlO}_{3}$ at 1 ps and $T=$ $10 \mathrm{~K}$. The metallic-state conductivity value $\sigma_{\mathrm{dc}}(T=400 \mathrm{~K})$ was used to fit the $\mathrm{SmNiO}_{3}$ layer, while all phonon parameters were kept unchanged for the $\mathrm{LaAlO}_{3}$ substrate. Similar to the equilibrium case [10], $\mathrm{SmNiO}_{3}$ is a bad metal in the transient state. The real part of the optical conductivity shows a broad Drude peak with a scattering rate $1 / \tau=1000 \mathrm{~cm}^{-1}$ [Fig. 3(b)]. Similar fits were done for other time delays. The extracted zero-frequency conductivity of $\mathrm{SmNiO}_{3}$ is shown as dark-blue squares in Fig. 3(c). The data at $-1 \mathrm{ps}$ is the equilibrium value, calculated from the resistivity [Fig. 1(c)]. The error bars are standard deviations from various experimental runs. The mean value of the transient conductivity increases to $2190 \Omega^{-1} \mathrm{~cm}^{-1}$ within $1.5 \mathrm{ps}$, and then decays exponentially back to the equilibrium value. According to the Drude model, the zero-frequency conductivity is $\sigma_{d c}=n e^{2} \tau / m^{*}$, where $n$ is the carrier density, $1 / \tau$ is the scattering rate and $m^{*}$ is the carrier effective mass. For all pump-probe time delays, our fit shows a constant Drude width (i.e., a constant scattering rate).
Assuming that the carrier effective mass does not change in the light-induced state, we relate the increase in the zero-frequency conductivity to an increase in carrier density.

Figure 3(c) displays the fitted transient conductivity for different base temperatures. The maximum transient
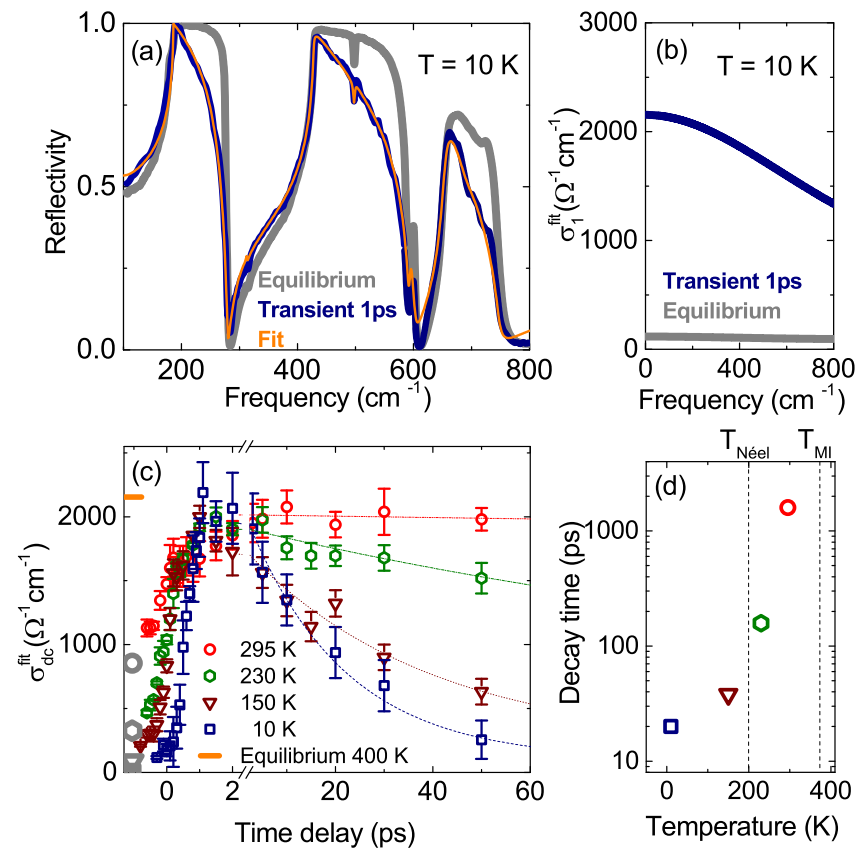

FIG. 3. (a) Equilibrium reflectivity (gray), transient reflectivity at 1 ps (dark blue), and the fit (orange) of $\mathrm{SmNiO}_{3} / \mathrm{LaAlO}_{3}$. (b) The real part of the optical conductivity extracted from the fit. (c) Within experimental error, the same maximum transient conductivity is reached for all measured temperatures. (d) The lifetime of the transient state tends to diverge approaching the metal-insulator transition temperature $T_{M I}$. 
conductivities reach the same level within the experimental error, independent of whether the starting equilibrium phase is an antiferromagnetic insulator $(T=10$ and $150 \mathrm{~K})$ or a paramagnetic insulator $(T=230$ and $295 \mathrm{~K})$. Consistently, the lifetime of the transient state did not show any correlation with the antiferromagnetic ordering temperature [Fig. 3(d)], increasing continuously with increasing temperature and smoothly crossing the Néel temperature. This lifetime was observed to diverge close to the metal-insulator transition, as expected for the decay time close to the critical point.

Because neither the transient conductivity or the lifetime of the transient state show anomalies at the equilibrium Néel temperature, we conclude that the light-induced effect proceeds independently of preexisting magnetic order. The physics of the interface control may then be driven either by a structural phase transition launched in the material or by a purely electronic mechanism, for example, fast melting of charge order. The present data cannot resolve this problem.

Our data offer insight into understanding the role of the driven substrate lattice in triggering the insulator-metal transition in the nickelate. No phonon splitting or frequency shifting for the substrate phonons is observed in the transient state, which rules out possible symmetry changing in the substrate. Contrary to the $20 \%$ reflectivity drop at around $700 \mathrm{~cm}^{-1}$ with increasing temperature [Fig. 1(a)], there was no observable laser heating in the substrate [Figs. 2(a)-2(c)], which excludes the possibility that a thermally induced strain modification may trigger the insulator-metal transition in the nickelate. The reflectivity reshaping at the transverse plasma frequency of the driven substrate phonon indicates the substrate as a transducer only at early time delays, driving the transient insulator-metal transition in the $\mathrm{SmNiO}_{3}$ film.

In summary, we have demonstrated a transient conductivity enhancement in the insulating $\mathrm{SmNiO}_{3}$ thin film when the substrate phonon is driven by midinfrared pulses. This is reminiscent of the effect observed in $\mathrm{NdNiO}_{3}$ films [3]. The transient state in $\mathrm{SmNiO}_{3}$ has the same carrier density as the equilibrium high-temperature metallic phase, despite negligible heating. The maximum transient conductivity reaches the same metallic-state value, starting from the antiferromagnetic and paramagnetic insulating phases. This indicates that the light-induced insulator-metal transition is not driven by the melting of the magnetic order. More generally, our work offers insight into understanding and further exploring the control of heterointerfaces with light.

\section{ACKNOWLEDGMENTS}

We thank Stefan Kaiser, Roberto Merlin, Miroslav Abrashev, Stefan Zollner, Alaska Subedi, Oleg E. Peil, Roman Mankowsky, and Michael Först for extensive discussions. This research has received financial support from the European Research Council under the European Union's Seventh Framework Programme Grant Agreement No. 319286 (Q-MAC). Part of this work was supported by the Swiss National Science Foundation through Division II.
[1] J. Liu, M. Kargarian, M. Kareev, B. Gray, P. J. Ryan, A. Cruz, N. Tahir, Y.-D. Chuang, J. Guo, J. M. Rondinelli, J. W. Freeland, G. A. Fiete, and J. Chakhalian, Nat. Commun. 4, 2714 (2013); M. Hepting, M. Minola, A. Frano, G. Cristiani, G. Logvenov, E. Schierle, M. Wu, M. Bluschke, E. Weschke, H.-U. Habermeier, E. Benckiser, M. Le Tacon, and B. Keimer, Phys. Rev. Lett. 113, 227206 (2014); A. J. Hauser, E. Mikheev, N. E. Moreno, J. Hwang, J. Y. Zhang, and S. Stemmer, Appl. Phys. Lett. 106, 092104 (2015).

[2] S. Catalano, M. Gibert, V. Bisogni, O. E. Peil, F. He, R. Sutarto, M. Viret, P. Zubko, R. Scherwitzl, A. Georges, G. A. Sawatzky, T. Schmitt, and J.-M. Triscone, APL Mater. 2, 116110 (2014).

[3] A. D. Caviglia, R. Scherwitzl, P. Popovich, W. Hu, H. Bromberger, R. Singla, M. Mitrano, M. C. Hoffmann, S. Kaiser, P. Zubko, S. Gariglio, J.-M. Triscone, M. Först, and A. Cavalleri, Phys. Rev. Lett. 108, 136801 (2012).

[4] M. Först, A. D. Caviglia, R. Scherwitzl, R. Mankowsky, P. Zubko, V. Khanna, H. Bromberger, S. B. Wilkins, Y.-D. Chuang, W. S. Lee, W. F. Schlotter, J. J. Turner, G. L. Dakovski, M. P. Minitti, J. Robinson, S. R. Clark, D. Jaksch, J.-M. Triscone, J. P. Hill, S. S. Dhesi, and A. Cavalleri, Nat. Mater. 14, 883 (2015).

[5] M. K. Stewart, J. Liu, M. Kareev, J. Chakhalian, and D. N. Basov, Phys. Rev. Lett. 107, 176401 (2011).
[6] Z. M. Zhang, B. I. Choi, M. I. Flik, and A. C. Anderson, J. Opt. Soc. Am. B 11, 2252 (1994).

[7] G. Catalan, Phase Transit. 81, 729 (2008); M. L. Medarde, J. Phys.: Condens. Matter 9, 1679 (1997).

[8] M. V. Abrashev, A. P. Litvinchuk, M. N. Iliev, R. L. Meng, V. N. Popov, V. G. Ivanov, R. A. Chakalov, and C. Thomsen, Phys. Rev. B 59, 4146 (1999).

[9] J. Ruppen, J. Teyssier, O. E. Peil, S. Catalano, M. Gibert, J. Mravlje, J. M. Triscone, A. Georges, and D. van der Marel, Phys. Rev. B 92, 155145 (2015).

[10] R. Jaramillo, S. D. Ha, D. M. Silevitch and S. Ramanathan, Nature Phys. 10, 304 (2014).

[11] Since commercial $\mathrm{LaAlO}_{3}$ samples are twinned, both the bare substrate and the nickelate heterostructure show isotropic optical response.

[12] I.-C. Ho, X. Guo, and X.-C. Zhang, Opt. Express 18, 2872 (2010).

[13] R. Huber, A. Brodschelm, F. Tauser, and A. Leitenstorfer, Appl. Phys. Lett. 76, 3191 (2000).

[14] W. Zhong, R. D. King-Smith, and D. Vanderbilt, Phys. Rev. Lett. 72, 3618 (1994).

[15] M. Dressel and G. Grüner, Electrodynamics of Solids (Cambridge University Press, Cambridge, UK, 2002). 